\title{
Association between coronary heart disease and chronic oral infections
}

\author{
Sujatha S Reddy로 Sri Rekha², Aninditya Kaur³, Poornima E ${ }^{4}$
}

Quick Response Code

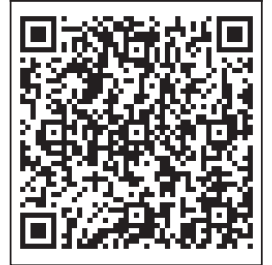

doi: $10.5866 / 2015.7 .10090$

1Professor

${ }^{3 \& 4}$ Post Graduate Student

Dept of Oral medicine, Diagnosis and Radiology, M.S.Ramaiah Dental college \& Hospital, MSRIT Post, New BEL Road, Bangalore-560054. Karnataka India ${ }^{2}$ Asst Professor, Dept of Oral medicine, Diagnosis and Radiology, G. Pulla Reddy Dental College, Kurnool, Andhra Pradesh, India

\section{Article Info:}

Received: April 9, 2015

Review Completed: May 10, 2015

Accepted: J une 10, 2015

Available Online: April, 2015 (www.nacd.in)

(c) NAD, 2015 - All rights reserved

\section{Email for correspondence:}

s_sujathajanardhan@yahoo.com

\begin{abstract}
:
BACKGROUND: Coronary Heart Disease (CHD) has a complex aetiology and is associated with a strong genetic component, age, gender and environmental factors. Chronic oral infection contributes to chronic systemic inflammatory burden causing CHD occurrence and progression.
\end{abstract}

OBJ ECTIVE: To investigate and compare the various serum inflammatory markers among CHD patients and non-CHD controls; and to determine if a link exists between these markers and oral health status.

MATERIALS AND METHODS: A total of 55 patients and 55 healthy individuals were included in the study. The subjects were explained about the procedure and consent was obtained. Blood pressure, glucose level, HbA 1c, hemoglobin, LDL cholesterol, leucocytes, fibrinogen, CPK, C-reactive protein serum levels were analysed. Oral exami nation was carried out which included number of teeth, missing and decayed teeth, filled teeth, endodontically treated teeth. A set of standard periodontal parameters (probing pocket depths, bleeding on probing (BOP), clinical attachment level, periodontal screening index (PSI) recorded.

RESULTS: Patients with CHD exhibited an unfavourable oral health status, an increase in number of missing and decayed teeth was observed. A significant relationship between increasing severity of periodontitis (PSI) with the mean of 2.254 patients with CHD and 1.688 in normal subjects was observed. A statistically significant difference was observed in fibrinogen, CPK, CRP, LDL, blood glucose, leucocytes levels in patients with CHD and controls. CONCLUSION: Low gradechronic inflammation plays an important role as a pathogenic determinant in the development of CHD. Patients with CHD had an unfavourable oral heal th suggesting that chronic inflammation can be a possible risk factor of CHD.

Key words: Coronary heart disease, periodontitis, endodontic lesions, C-reactive protein (CRP), fibrinogen

\section{INTRODUCTION:}

Coronary heart disease (CHD) is epidemic in India and is one of the major causes of death with $40 \%$ of the deaths occurring in urban areas and $30 \%$ in rural areas. ${ }^{1}$ Besides the well-established risk factors for $\mathrm{CHD}$ such as smoking hypertension, high low density lipoprotein (LDL) serum levels, diabetes, gender, obesity, socioeconomic status and genetic dispositions, chronic inflammatory processes have 
been considered as potential predictors for arteriosclerosis. ${ }^{2}$ The situation is further compounded by the fact that periodontal disease is highly prevalent in India, and it can act as an independent risk factor for CHD. By far periodontal disease is the most common oral infection in India, with a prevalence rate of $66.2 \%$ among individuals of age 15 years and about $89.2 \%$ among adults in the age group of 35-44 years. ${ }^{3}$ Common risk factors exist for both the diseases such as age, gender, alcohol, tobacco, diet, socioeconomic status, hypertension, diabetes, stress etc.

Periodontal disease is predominantly a Gramnegative infection resulting in severe inflammation, with potential in vascular dissemination via the sulcular epithelium of microorganisms and their products such as Lipopolysaccharides (LPS) throughout the body. The severity of the inflammation determines the degree of bacteremia. Oral infections like dental caries and periodontitis are known not only to enhance the risk of certain systemic diseases like coronary heart disease and related events such as angina, infarction and atherosclerosis; but also stroke; diabetes mellitus; preterm labour; and respiratory conditions such as Chronic Obstructive Pulmonary Disease (COPD). ${ }^{4,5}$

Significant association between poor oral health, chronic oral inflammations, and CHD has been pointed out in few studies proving the important role played by inflammatory and hemostatic factors. ${ }^{6}$ The secretion of proinflammatory cytokines from the vascular endothelium as well as from macrophages induces the production of inflammatory molecules that are measured in the circulation, such as C-reactive protein (CRP), and fibrinogen. ${ }^{7} \mathrm{High}$ levels of fibrinogen and coagulation factor VII are associated with an increased risk of CHD. Factor VII levels are higher in individuals with a high intake of dietary fat. Decreased fibrinolytic activity has been reported in patients with coronary atherosclerosis. Elevated levels of serum CRP, high levels of Lipoprotein (a) a compound formed from LDL cholesterol, high blood triglycerides are associated with an increased risk of CHD events. ${ }^{8}$ Given the high prevalence of oral infections especially periodontal disease and its deleterious association with coronary heart disease it is essential to prove that a baseline oral disease is significantly associated with an increased risk of CHD. Hence the present study was undertaken to identify certain serum markers with higher specificity for CHD

\section{MATERIAL AND METHODS}

Fifty five patients between the age group of 40 to 80 years with the history of Ischemic heart disease(IHD), Congestive cardiac failure (CCF), Myocardial infarction (MI), Angina pectoris, as confirmed with ECG changes and certain serum enzyme levels like glutamic oxaloacetic acid, creatinine, phosphokinase, visiting the Department of Cardiology, formed our study group. Age matched 55 subjects with good general health (age, ethnicity) without clinical evidence of cardiovascular disease as confirmed by a cardiologist at least 6 months prior to the oral examination formed the control group. An ethical clearance was obtained from the Institutional review board and informed consent was obtained from all subjects. Among patients who were deemed clinically stable to visit the dental hospital and undergo a thorough oral examination were included in the study. Exclusion criteria were completely edentulous patients or patients having less than 5 teeth in oral cavity, those who had received periodontal treatment within the preceding 6 months to the disease and cardiac patients on treatment. Age, gender height and weight, BMI (body mass index) were recorded. All subjects were asked for family history of coronary artery disease, tobacco habits, blood pressure and diabetes mellitus. Tobacco use was assessed as current, former or never, type of tobacco (smoking/smokeless) and duration and frequency of tobacco use was recorded. Blood pressure, glucose level, $\mathrm{HbAlc}$ (glycosylated haemoglobin), Low Density Lipoprotein (LDL) cholesterol, leucocytes, fibrinogen, creatine phosphokinase (CPK), C-reactive protein (CRP) serum levels and ESR levels were observed. Glucose levels and $\mathrm{HbAlc}$ lcerved as an indicator for diabetes status. 
Oral examination, all subjects were required to complete a questionnaire and undergo oral examination by two examiners for number of teeth, missing and decayed teeth, filled teeth, endodontically treated teeth, bleeding on probing, clinical attachment level, PSI (Periodontal Screening Index). PSI serves as a valuable tool to obtain information about the periodontal pathology and treatment needs and help to distinguish between clinically healthy and inflamed sites. All measurements were recorded at 6 aspects on each of 6 Ramjford teeth (mesiobuccal, mid-buccal, distobuccal, mesiolingual, midl-lingual and disto lingual) by using a standard periodontal probe (Williams probe) and non-magnifying dental mirror. Screening index ranges from degree $0-4$. Score 0 - no bleeding on probing, no pathologic pocket, and no calculus. Score 1 - bleeding on probing, Score 2 calculus and no pathol ogical pocket, Score 3 - probing depth between 3.5 - $5.5 \mathrm{~mm}$, Score 4 - probing depth $>5.5 \mathrm{~mm}$.

\section{Statistical analysis:}

Statistical analysis was performed by mean of SPSS 15.0 for Windows software. Descriptive statistics comprising of mean and standard deviation was used for continuous data. Percentage and proportions were analysed for discontinuous data such as gender. Chi square test was used for comparing the statistical significant differences in proportions between different groups. Independent $T$ test was used to compare the statistical significance of mean (standard deviation) between 2 groups. $p<0.05$ was considered statistically significant.

\section{RESULTS}

Evidence of an association between chronic dental health and CHD was found in the present study. CHD patients were in the mean age of 59.4 years (SD 8.3 years) and a distribution of $69.1 \%$ males and $30.9 \%$ females (Table 1); the controls had a mean age of 57.8 years (SD 8.5 years) and a distribution of $56.4 \%$ males and $43.6 \%$ females (Table 1). Patients with CHD were generally overweight $(n=37,67.2 \%)$, and some obese $(n=14$, $25.4 \%)$. Most of them were Diabetics and had increased BP. Also there was statistically significant difference between controls and CHD patients in fibrinogen CPK, CRP, LDL, Blood glucose, Leucocytes levels (Table II). In comparison to controls, CHD patients had an unfavourable dental status, a statistically significant number of missing teeth and decayed teeth were present. There was no significant difference for filled teeth between both groups. Normal controls showed higher number of endodontically treated teeth than patients with CHD. A significant relationship between increasing severity of periodontics (PSI) with the mean of 2.254 in patients with CHD and 1.688 in normal subjects was observed (Table III).

\section{DISCUSSION}

A strong genetic component is implicated in the genesis of atherosclerosis, the most common cause of coronary heart disease (CHD) in association with several anatomical, physiological, and behavioural risk factors, including changes in serum lipid profile, smoking, arterial hypertension, diabetes, obesity, sedentary lifestyle, age, and gender. Many cell types, including platelets, endothelial cells, activated monocytes, macrophages, and smooth muscle cells, are involved in the formation of atherosclerotic plaques. Certain cardiovascular (CV) risk markers such as low-grade chronic inflammation, at both focal and systemic levels, play a key role in destabilization and rupture of atherosclerotic plaques, leading to acute cardiovascular (CV) events. ${ }^{9-11}$

An association of serological markers of bacterial and viral infections and biochemical markers of inflammation with cardiovascular disease has been found in few studies. ${ }^{12}$ It has even been suggested that atherosclerosis is primarily an inflammatory disease. A large number of inflammatory serum biomarkers are associated with the risk of CHD in prospective studies like CRP,LpPLA2., white blood cell counts, myeloperoxidase, serum phospholipase A2 (sPLA2), cystatins, 
Table I: Distribution of Gender in the study population

\begin{tabular}{lcccccc} 
& \multicolumn{2}{c}{ NORMAL CONTROLS } & & \multicolumn{2}{c}{ CHD } \\
\cline { 2 - 3 } Gender & Frequency & & Percent & & Frequency & Percent \\
Male & 31 & 56.4 & & 38 & 69.1 \\
\hline Female & 24 & 43.6 & & 17 & 30.9 \\
\hline Total & 55 & 100.0 & & 55 & 100.0 \\
\hline
\end{tabular}

Table II: Group Characteristics in the study population

\begin{tabular}{|c|c|c|c|c|c|}
\hline & Group & $\mathbf{N}$ & Mean & Std Deviation & p Value \\
\hline \multirow[t]{2}{*}{ Age } & Normal & 55 & 57.80 & 8.486 & $>.005$ \\
\hline & $\mathrm{CHD}$ & 55 & 59.47 & 8.302 & $>.005$ \\
\hline \multirow[t]{2}{*}{ BMI } & Normal & 55 & 21.02 & 3.100 & $>.005$ \\
\hline & $\mathrm{CHD}$ & 55 & 22.53 & 2.956 & $>.005$ \\
\hline \multirow[t]{2}{*}{ PSI } & Normal & 55 & 1.688 & .5142 & $<001$ \\
\hline & $\mathrm{CHD}$ & 55 & 2.254 & .7749 & $<001$ \\
\hline \multirow[t]{2}{*}{ SYSBP } & Normal & 55 & 124.07 & 8.537 & $<001$ \\
\hline & $\mathrm{CHD}$ & 55 & 135.36 & 17.382 & $<001$ \\
\hline \multirow[t]{2}{*}{ DASBP } & Normal & 55 & 92.29 & 10.025 & $>.005$ \\
\hline & $\mathrm{CHD}$ & 55 & 92.56 & 11.908 & $>.005$ \\
\hline \multirow[t]{2}{*}{$\mathrm{Hb}$} & Normal & 55 & 13.84 & 1.515 & $<001$ \\
\hline & $\mathrm{CHD}$ & 55 & 12.80 & 1.505 & $<001$ \\
\hline \multirow[t]{2}{*}{ Glucose Level } & Normal & 55 & 90.71 & 10.640 & $<001$ \\
\hline & $\mathrm{CHD}$ & 55 & 137.27 & 49.984 & $<001$ \\
\hline \multirow[t]{2}{*}{ LDL } & Normal & 55 & 117.29 & 9.253 & $<005$ \\
\hline & $\mathrm{CHD}$ & 55 & 107.81 & 20.258 & $<005$ \\
\hline \multirow[t]{2}{*}{ Fibrinogen } & Normal & 55 & 296.89 & 58.191 & $<001$ \\
\hline & $\mathrm{CHD}$ & 55 & 484.67 & 139.726 & $<001$ \\
\hline \multirow[t]{2}{*}{ CPK } & Normal & 55 & 109.71 & 33.448 & $<001$ \\
\hline & $\mathrm{CHD}$ & 55 & 254.29 & 162.825 & $<001$ \\
\hline \multirow[t]{2}{*}{ CRP } & Normal & 55 & 35.42 & 39.093 & $<001$ \\
\hline & $\mathrm{CHD}$ & 55 & 96.59 & 96.661 & $<001$ \\
\hline \multirow[t]{2}{*}{ Leucocytes } & Normal & 55 & 7107.60 & 1445.329 & $>.005$ \\
\hline & $\mathrm{CHD}$ & 55 & 10825.81 & 9748.209 & $>.005$ \\
\hline
\end{tabular}

Table III: Frequency of Missing teeth, Decayed teeth, Filled teeth and Endodontically treated teeth in the study population

\begin{tabular}{lcccc} 
& Missing teeth & Decayed Teeth & Filled teeth & $\begin{array}{c}\text { Endodontically } \\
\text { treated teeth }\end{array}$ \\
Normal Controls & 23 & 27 & 9 & 14 \\
\hline CHD & 37 & 32 & 10 & 14 \\
\hline
\end{tabular}


fibrinogen, interleukins etc. Dental infections have been associated with CHD. Periodontal disease is a chronic infection of the supporting tissues of the tooth and apical periodontitis, a consequence of dental caries involving the pulp have demonstrated the possible relationship between chronic dental infection and CVD. Consequently, the relationship between oral disease and systemic disease is a twoway road, with systemic host factors acting locally to reduce resistance to periodontal destruction and the local bacterial challenge generating widespread effects with the potential to induce adverse systemic outcomes. Chronic infections of dental origin are cumulative in patients with CHD and have been evaluated in various studies. ${ }^{13-15}$

In the present study most of the patients were either overweight or obese with the Body Mass Index (BMI) ranging between 25 and 32. Erythrocyte sedimentation rates levels were elevated in most patients which could be because of high proportion of fibrinogen in the blood which causes red blood cells to stick to one other forming stacks called 'rouleaux,' which settle faster. Inflammatory markers like serum Fibrinogen and C-reactive protein concentrations were higher in the $\mathrm{CHD}$ group similar to other studies. ${ }^{12,16} \mathrm{Fibrinogen}$ increases therisk of an acute coronary event through its prothrombotic and rheological effects, and may also play a part in atherosclerosis formation. ${ }^{17} \mathrm{CRP}$ levels often increase substantially in response to a wide variety of biological insults, infections, inflammatory conditions and malignant processes. ${ }^{18,19}$ However, given its consistent association with increased CHD risk, CRP remains an established 'marker of risk' its increased levels are associated with increased CHD risk. In the absence of inflammation, hsCRP levels of, $1 \mathrm{mg} / \mathrm{ml}$ confer a lower risk for CHD, while levels above 3 $\mathrm{mg} / \mathrm{ml}$ increase the risk of CHD.

Periodontal diseases are associated with an increase in CRP levels, higher levels in generalized periodontitis compared to localized periodontitis. In our study, dentate people with extensive periodontal disease had an increase of approximately one-third in mean CRP and a doubling in the prevalence of elevated CRP, compared with periodontaly healthy people. ${ }^{20}$ Individuals with periodontal disease have an approximately $24-35 \%$ increase in risk of developing CHD. Periodontal disease also may lead to transient increase in circulating levels of IL-I $\beta$, TNF- $\alpha$, and prostaglandin $\mathrm{E}_{2}\left(\mathrm{PGE}_{2}\right)$. This may be the first step in the contribution of periodontal diseases to systemic inflammation. Elevated levels of CRP, IL-6 and neutrophils in patients with periodontitis may occur when bacteria, bacterial products and cytokines enter the circulation. ${ }^{21,22}$ In a large epidemiologic survey it was observed that chronic periodontal disease was associated with an increased risk of CHD. Elevated levels of CRP, fibrinogen, serum amyloid A, and von Willebrand's factor were correlated with periodontal disease. Likewise, the number of missing teeth has also been linked with elevated risk levels for CHD .A relationship between oral health and CHD was demonstrated in this long study of 14 years where edentulous individuals or patients with periodontal diseases exhibited a higher risk for CHD in $25 \%$, and male patients with periodontal diseases exhibited higher risks for $\mathrm{CHD}$ in $70 \%{ }^{23}$

Besides periodontal disease, chronic apical inflammations of endodontic origin could be considered as a possible source of bacteraemia. Although there are differences between chronic inflammatory disease of periodontal and endodontic origin, there are similarities regarding common existing microbiota and systemic cytokine levels. ${ }^{6}$ In the study by J oshipura et al a modest association between root canal therapy following pulp inflammation and incidence of CHD was observed. ${ }^{12}$

Large number of patients had el evated glucose and $\mathrm{HbAlc}$ levels indicating their diabetic status. Cholesterol (LDL) was higher among CHD patients in accordance to other studies where it has been strongly associated with coronary heart disease (CHD) risk. Even at concentrations well below the National Cholesterol Education Program target of $130 \mathrm{mg} / \mathrm{dL}$, LDL cholesterol is a strong independent predictor of coronary heart disease in individuals 
with diabetes. Hence, aggressive control of LDL cholesterol in individuals with poor glycaemic control with a target level of $<100 \mathrm{mg} / \mathrm{dL}$ is recommended. ${ }^{24}$ Creatine phospokinase (CPK) a myocardial enzyme, an inflammatory biomarker was also significantly elevated in our study. Leukocyte count was significantly elevated among CHD patients. It is the most sensitive and the most specific indicator available for the diagnosis of an acutemyocardial infarction, the degreeand duration of CPK elevation in serum approximates the extent of an acute myocardial infarction. ${ }^{25}$

In the present study the mean leukocyte count was higher in patients than in control subjects 8,000/ $\mathrm{mm}$ vs. $7,500 / \mathrm{mm}$ similar to other studies. ${ }^{26}$ Leukocyte count is a marker of inflammation. Elevated differential cell counts of various types of inflammatory cells, including eosinophil, neutrophil, and monocyte counts, also predict the future incidence of CHD. Numerous epidemiologic and clinical studies have shown leukocytosis to be an independent predictor of future cardiovascular events, both in healthy individuals free of CHD at baselineand in patients with stable angina, unstable angina, or a history of myocardial infarction. Leukocytosis affects CHD through multiple pathologic mechanisms that mediate inflammation, cause proteolytic and oxidative damage to the endothelial cells, plug the microvasculature, induce hypercoagulability, and promoteinfarct expansion. ${ }^{27}$ The leukocyte count is an inexpensive, reliable, easy to interpret, and ordered routinely, however, its diagnostic and prognostic utility in CHD is widely unappreciated.

In the present study, CHD patients had significantly less number of teeth, but large number of endodontic and periodontal lesions in the remaining teeth compared to the control group. PSI score was high. Most teeth needed either a restoration or a root canal therapy. These findings are in accordance with other investigations in which the association between CHD, physical health, socioeconomic status, and poor oral health has been demonstrated. ${ }^{28-30} \mathrm{~A}$ possible relationship between lesions of endodontic origin and CHD was also evaluated in a study by Caplan et al who found that patients younger than 40 years exhibited a positive correlation between endodontic lesions and diagnosis of CHD, however, individuals older than 40 years showed no statistically significant association. ${ }^{27}$ The assumptions that chronic infections are involved in the pathogenesis of $\mathrm{CHD}$ and that chronic infections of dental origin are cumulative in patients with $\mathrm{CHD}$ have been observed in different prospective studies. ${ }^{12,} 30$

\section{CONCLUSION}

Periodontal disease is one of the risk factors for cardiovascular diseaseand possibly one of its causes. Hence, even associations of modest magnitude have a large impact. Oral infections are treatable; moreover, it is preventable. Oral disease prevention and promotion of oral health need to be integrated with chronic disease prevention and general health promotion as the modifiable risk factors like tobacco use, oral hygiene, diet etc are linked. Hence, effective integration of oral disease management with management of other chronic diseases is essential.

\section{References}

1. Gupta R. Recent trends in coronary heart disease epidemiology in India. Indian Heart J 2008; 60(2):4-18.

2. Anderson KM, Castelli WP, Levy D. Cholesterol and mortality. 30 years of follow-up from the $F$ ramingham study. J AMA 1987; 257:2176-2180.

3. National Oral Health Survey and Flouride Mapping: Dental Council of India. New Delhi: Dental Council of India; 2004. [Last accessed on 2010 Mar 10]. pg 13. Available from: http:/ /iaphd.org/NationalSurvey/summary.pdf.

4. Page RC, Beck J D. Risk assessment for periodontal diseases. Int Dent J 1997; 47:61-65.

5. Arpita Gur and J P Majra. Awareness Regarding the Systemic Effects of Periodontal Disease among Medical Interns in India. J Glob Infect Dis. 2011; 3(2):123-127.

6. Brita Willershausen, Adrian Kasaj, Ines Willershausen, Denisa Zahorka, Benjamin Briseno, Maria Blettner,Sabine Genth-Zotz, Thomas Munzel. Association between Chronic Dental Infection and Acute Myocardial Infarction. J OE 2009; 35(5).

7. Nikolaos Papageorgiou, Dimitris Tousoulis, Gerasimos Siasos, Christodoul os Stefanadis. Is Fibrinogen a Marker of Inflammation inCoronary Artery Disease? Hellenic J Cardiol 2010; 51: 1-9 
8. Mohammad Madjid and J ames T. Willerson .I nflammatory markers in coronary heart disease. British Medical Bulletin 100(1):23-38.

9. Stollberger C, Finsterer J. Role of infectious and immune factors in coronary and cerebrovascular arteriosclerosis. Clin Diagn Lab I mmunol 2002; 9(2):207-215.

10. Naoum J J, Chai H, Lin PH, Lumsden AB, Yao Q, Chen C. Lymphotoxin-? and cardiovascular disease: clinical association and pathogenic mechanisms. Med Sci Monit 2006; 12(7):121-124.

11. Libby P. Inflammation in atherosclerosis. Nature 2002; 420:868-874.

12. Koenig W, Sund M, Fröhlich M, et al. C-reactive protein, a sensitive marker of inflammation, predicts future risk of coronary heart disease in initially healthy middle-aged men. Results from the MONICA (Monitoring trends and determinants in cardiovascular disease) Augsburg Cohort study, 1984 to 1992. Circulation 1999; 99:237-242.

13. J oshipura KJ, Pitiphat W, Hung HC, Willett WC, Colditz GA, Douglass CW. Pulpal inflammation and incidence of coronary heart disease. J Endod 2006; 32:99-103.

14. Kinane DF. Periodontal diseases contributions to cardiovascular disease: an overview of potential mechanisms. Ann Periodontol 1998; 3:142-150.

15. Larkin M. Link between gum disease and heart disease disputed. Lancet 2001; 358.

16. J ukka H. Meurman, Sok-J a J anket, Markku Qvarnström, Pekka Nuutinen. Dental infections and serum inflammatory markers in patients with and without severe heart disease. Oral Surg Oral Med Oral Path Oral Rad End 2003; 96(6):695-700.

17. Ernst E, Resch K. Fibrinogen as cardiovascular risk factor: a meta-analysis and review of the literature. Ann Intern Med 1993; 118:956-963.

18. Madjid M, Casscells S, Willerson J T. Inflammatory biomarkers as surrogate markers in detection of vulnerable plaques and vulnerable patients. In: Willerson J T (ed). Cardiovascular Medicine, 3rd edn. Springer, London, UK. 2007, 641-651.

19. Pasceri V, Willerson J T, Yeh ET. Direct proinflammatory effect of C-reactive protein on human endothelial cells. Circulation 2000; 102:2165-2168.
20. Slade GD, Offenbacher S, Beck J D, Heiss G, Pankow J S. Acute-phase inflammatory response to periodontal disease in the US population. J Dent Res 2000; 79(1):49-57

21. Renuka Devi Ramamoorthy, Vijaykumar Nallasamy, Raghavendra Reddy, Nalini Esther, Yuvaraja Maruthappan. A review of C-reactive protein: A diagnostic indicator in periodontal medicine. J of Pharmacy Bioallied sciences. Dental science- review article. 2012; 4(6):422-426.

22. Dhruva Kumar Gani, Deepa Lakshmi, Rama Krishnan, and Pamela Emmadi. Evaluation of C-reactive protein and interleukin- 6 in the peripheral blood of patients with chronic periodontitis. J Indian Soc Periodontol 2009; 13(2):69-74.

23. Mattila $K$, Vesanen $M$, Valtonen $V$, Nieminen $M$, Paiosuo $T$, Rasi $V$, et al. Effect of treating periodontitis on C-reactive protein levels: A pilot study. BMC Infect Dis 2002; 2:30.

24. Richard B. Devereux, Linda D. Cowan, R. Stuart Gray, Thomas K. Welty, Oscar T. Go and Barbara V. Howard et al. With Insulin Resistance and Low LDL: TheStrong Heart Study LDL Cholesterol as a Strong Predictor of Coronary Heart Disease in Diabetic Individuals. Arterioscler Thromb Vasc Bio 2000; 20:830-835.

25. Peter M. Guzy, MD, PhD, Fellow in the Division of Cardiology, Robert Wood J ohnson. Creatine PhosphokinaseMB (CPK-MB) and the Diagnosis of Myocardial Infarction. West J Med 1977; 127(6):455-460.

26. G.D. Friedman, A.L. Klatsky, A.B. Siegelaub.The leukocyte count as a predictor of myocardial infarction. N Engl J Med 1974; 290:1275-1278.

27. Caplan DJ , Chasen J B, Krall EA, et al. Lesions of endodontic origin and risk of coronary heart disease. J Dent Res 2006; 85:996-1000.

28. Cueto A, Mesa F, Bravo M, Ocan a-Riola R. Periodontitis as risk factor for acute myocardial infarction: a case control study of Spanish adults. J Periodont Res 2005; 40:36-42.

29. J anket SJ , Baird AE, Chuang SK, J ones J A. Meta-analysis of periodontal disease and risk of coronary heart disease and stroke. Oral Surg Oral Med Oral Pathol Radiol Endod 2003; 95:559-569.

30. Grau AJ , Becher H, Ziegler CM, et al. Periodontal disease as a risk factor for ischemic stroke. Stroke 2004; 35: 496-501.

\section{Gain quick access to our journal online View our journal at www.nacd.in}

